\title{
Association between variants in the genes for adiponectin and its receptors with insulin resistance syndrome (IRS)-related phenotypes in Mexican Americans
}

\author{
D. K. Richardson • J. Schneider • M. J. Fourcaudot • \\ L. M. Rodriguez $\cdot$ R. Arya $\cdot$ T. D. Dyer $\cdot$ L. Almasy $\cdot$ \\ J. Blangero • M. P. Stern • R. A. DeFronzo • \\ R. Duggirala $\cdot$ C. P. Jenkinson
}

Received: 19 December 2005 / Accepted: 12 June 2006 / Published online: 29 August 2006

(C) Springer-Verlag 2006

\begin{abstract}
Aims/hypothesis The aim of this study was to examine whether genetic variation in $A D I P O Q, A D I P O R 1$ and $A D I P O R 2$ may contribute to increased susceptibility to components of the insulin resistance syndrome (IRS). Materials and methods We genotyped single-nucleotide polymorphisms (SNPs) in $A D I P O Q, A D I P O R 1$ and $A D I$ $P O R 2$ in Mexican American subjects $(N=439)$ and performed an association analysis of IRS-related traits.

Results Of the eight SNPs examined in the $A D I P O Q$ gene, rs4632532 and rs182052 exhibited significant associations with BMI ( $p=0.029$ and $p=0.032$ ), fasting specific insulin ( $p=0.023$ and $p=0.026)$, sum of skin folds (SS) $(p=0.0089$ and $p=0.0084$ ) and homeostasis model assessment of insulin sensitivity (HOMA- $\% \mathrm{~S})(p=0.015$ and $p=0.016$ ). Two other SNPs, rs266729 and rs2241767, were significantly associated with SS $(p=0.036$ and $p=0.013)$. SNP rs7539542 of ADIPOR1 was significantly associated with BMI, SS and waist circumference $(p=0.025, p=0.047$ and $p=0.0062$ ). Fourteen of the ADIPOR2 SNPs were found to
\end{abstract}

D. K. Richardson $(\bowtie) \cdot$ M. J. Fourcaudot $\cdot$ L. M. Rodriguez $\cdot$

R. A. DeFronzo $\cdot$ C. P. Jenkinson

Division of Diabetes MC 7886, Department of Medicine,

The University of Texas Health Science Centre at San Antonio,

7703 Floyd Curl Drive,

San Antonio, TX 78229-3900, USA

e-mail: richardsond2@uthscsa.edu

J. Schneider $\cdot$ T. D. Dyer $\cdot$ L. Almasy $\cdot$ J. Blangero $•$

R. Duggirala $\cdot$ C. P. Jenkinson

Southwest Foundation for Biomedical Research,

San Antonio, TX, USA

R. Arya $\cdot$ M. P. Stern

Division of Clinical Epidemiology, Department of Medicine,

University of Texas Health Science Centre at San Antonio,

San Antonio, TX, USA be significantly $(p<0.05)$ associated with fasting plasma triglyceride concentrations. Four of these SNPs (rs10848569, rs929434, rs3809266 and rs12342) were in high pairwise linkage disequilibrium $\left(r^{2}=0.99\right)$ and were strongly associated with fasting triglyceride levels ( $p=0.00029, p=0.00016, p=0.00027$ and $p=0.00021$ ). Adjusting for the effects of BMI and HOMA- $\% \mathrm{~S}$ on triglyceride concentrations increased significance to $p=0.000060$ for SNP rs929434. Bayesian quantitative trait nucleotide analysis was used to examine all possible models of gene action. Again, SNP rs929434 provided the strongest statistical evidence of an effect on triglyceride concentrations.

Conclusions/interpretation These results provide evidence for association of SNPs in $A D I P O Q$ and its receptors with multiple IRS-related phenotypes. Specifically, several genetic variants in ADIPOR2 were strongly associated with decreased triglyceride levels.

Keywords Adiponectin - Adiponectin receptors .

Association study - Genetics of type 2 diabetes mellitus .

Insulin Resistance Syndrome .

Single-nucleotide polymorphisms

$\begin{array}{ll}\text { Abbreviations } \\ \text { AMPK } & \begin{array}{l}\text { adenosine 5'-monophosphate-activated } \\ \text { protein kinase }\end{array} \\ \text { BQTN } & \begin{array}{l}\text { Bayesian quantitative trait nucleotide } \\ \text { fasting plasma glucose } \\ \text { FPG }\end{array} \\ \text { FSI } & \begin{array}{l}\text { fasting specific insulin } \\ \text { homeostasis model assessment of } \beta \text {-cell } \\ \text { HOMA- } \% \beta\end{array} \\ \text { HOMA-\%S } & \begin{array}{l}\text { homeostasis model assessment of insulin } \\ \text { sensitivity }\end{array} \\ \text { HWE } & \text { Hardy-Weinburg equilibrium }\end{array}$




$\begin{array}{ll}\text { IRS } & \text { insulin resistance (metabolic) syndrome } \\ \text { LD } & \text { linkage disequilibrium } \\ \text { LEP } & \text { fasting leptin } \\ \text { MAF } & \text { minor allele frequency } \\ \text { MGA } & \text { measured genotype approach } \\ \text { PAI-1 } & \text { plasminogen activator inhibitor-1 } \\ \text { SAFDS } & \text { San Antonio Family Diabetes Study } \\ \text { SNP } & \text { single-nucleotide polymorphism } \\ \text { SS } & \text { sum of skin fold thickness } \\ \text { TRL } & \text { triglyceride-rich lipoproteins } \\ \text { WC } & \text { waist circumference }\end{array}$

\section{Introduction}

Type 2 diabetes mellitus is a chronic metabolic disorder characterised by hyperglycaemia, insulin resistance, impaired beta cell function $[1,2]$ and a variety of cardiovascular risk factors, including hypertension, elevated triglycerides, reduced HDL cholesterol, increased plasminogen activator inhibitor-1 (PAI-1) levels, and endothelial dysfunction [3, 4]. Collectively, these metabolic and cardiovascular risk factors have been referred to as the insulin resistance syndrome (IRS) or metabolic syndrome. Insulin resistance in type 2 diabetes results from impaired insulin action in skeletal muscle and liver [5, 6]. Also, adipocytes are resistant to the anti-lipolytic action of insulin $[5,7]$, and the resultant increase in plasma NEFA exacerbates the insulin resistance in muscle and liver [5-9].

The prevalence of type 2 diabetes and the IRS is rapidly and relentlessly increasing and, although major risk factors for these disorders have been identified [1], the genetic factors responsible for type 2 diabetes mellitus and the IRS remain unclear. Genome-wide scans have demonstrated linkage between a number of chromosomal regions and diabetes or diabetes-related traits $[10,11]$. Candidate gene studies and a number of single-nucleotide polymorphisms (SNPs) have also been associated with type 2 diabetes and/or related diseases, including obesity and insulin resistance $[12,13]$.

Adiponectin (encoded by the gene $A D I P O Q$ ) is a hormone that is expressed only by adipocytes [14] and regulates energy homeostasis and glucose and lipid metabolism [15]. Rodent and human studies have demonstrated that adiponectin levels are decreased in insulin-resistant states, such as type 2 diabetes and obesity [16-19]. Administration of adiponectin to rodents with obesity and diabetes had insulin-sensitising effects [18] and reduced triglyceride accumulation in muscle [19]. Adiponectin acts through its receptors, ADIPOR1 and ADIPOR2. It was initially thought that $A D I P O R 1$ was primarily expressed in skeletal muscle, whereas ADIPOR2 was predominantly expressed in liver [20]. However, we recently showed that
ADIPOR2 is highly expressed in human muscle and may be the predominant isoform through which adiponectin exerts its insulin-sensitizing effects in skeletal muscle [21]. Adiponectin receptors mediate enhanced fatty acid oxidation and glucose uptake, increase adenosine 5'-monophosphate-activated protein kinase (AMPK) activity, and interact with peroxisome proliferator-activated receptor (PPAR) pathways [22, 23].

Common polymorphisms in the $A D I P O Q$ gene have been studied in several populations, including Japanese [24] and Europeans [25]. These studies suggest that genetic variation in the $A D I P O Q$ gene may predispose humans to insulin resistance and/or components of the IRS. Additionally, SNP +45 and SNP +276 polymorphisms of the $A D I P O Q$ gene have been shown to predict conversion from impaired glucose tolerance to type 2 diabetes in the Study to Prevent Non-Insulin-Dependent Diabetes Mellitus (STOP-NIDDM) [26]. In contrast, common ADIPOQ polymorphisms were not associated with type 2 diabetes in Pima Indians [27]. Studies of SNPs in the ADIPOR1 and $A D I P O R 2$ genes failed to find any association with type 2 diabetes in northern Europeans or African Americans [28], or in the Japanese population [29]. However, genetic variants in both $A D I P O R 1$ and ADIPOR2 genes were found to be associated with type 2 diabetes in the Old Order Amish population [30]. More recently, it was reported that variation in ADIPOR1 may affect insulin sensitivity and liver fat content in Europeans [31].

To date, no data have been published on the effects of genetic variation in the $A D I P O Q, A D I P O R 1$ and $A D I P O R 2$ genes on type 2 diabetes and the IRS in Mexican Americans, a group at high risk of these diseases [32]. In this study, we conducted a preliminary survey of these three candidate genes, selecting SNPs from the public SNP database (dbSNP) and from a review of the literature. We chose a subset of SNPs in the $A D I P O Q$ gene and all available SNPs in the recently discovered ADIPOR1 and ADIPOR 2 genes. We genotyped these common polymorphisms in a Mexican American population sample, and performed association analyses with IRS-related phenotypes.

\section{Subjects and methods}

\section{The San Antonio Family Diabetes Study}

All study participants were Mexican Americans from the San Antonio Family Diabetes Study (SAFDS) [10, 33]. Briefly, probands for the SAFDS were identified by diabetes status in an earlier epidemiological survey, the San Antonio Heart Study, which has been extensively described elsewhere [34]. All first, second and third degree relatives of the probands, aged from 18-98 years, were 
invited to participate in the study. Metabolic, anthropometric, demographic and medical history information was obtained on 439 individuals (116 with type 2 diabetes) distributed across 27 low-income Mexican American pedigrees [10, 33]. All procedures were approved by the Institutional Review Board of the University of Texas Health Science Centre at San Antonio, and all subjects gave informed consent prior to their participation.

For this study, we used phenotypic information from those subjects without diabetes ( $n=323$ individuals, depending on availability of data), to avoid metabolic derangements secondary to type 2 diabetes or treatment for type 2 diabetes. The following ten IRS-related phenotypes, which have been described in detail elsewhere [10], were considered for the present study: fasting plasma glucose (FPG), fasting specific insulin (FSI), homeostasis model assessment of $\beta$-cell function (HOMA- $\% \beta$ ) and insulin sensitivity (HOMA-\%S, an inverse measure of insulin resistance), fasting $\mathrm{HDL}$ cholesterol, fasting triglyceride concentrations, fasting leptin (LEP), BMI, waist circumference (WC) and sum of skin fold thickness (SS). The sum of eight skinfold measures was used as a measure of overall subcutaneous adiposity. Phenotype-specific distinct outliers were excluded from the analyses (e.g. triglyceride values $>9 \mathrm{mmol} / \mathrm{l})$. Where indicated, the data were log-transformed to normalise distributions.

\section{SNP genotyping}

Genomic DNA was isolated from whole blood (Qiagen, Chatsworth, CA, USA). SNPs were selected from the NCBI dbSNP database and from previous studies that reported association with diabetes, obesity and/or IRS-related traits. SNPs were obtained by either the Assay-on-Demand or Assay-by-Design service and genotyped according to manufacturer's instructions (Applied Biosystems, Foster City, CA, USA). Alleles were scored using the allelic discrimination software Sequence Detection System v2.1 (Applied Biosystems). All SNPs were tested for Mendelian pedigree inconsistencies. For all SNPs genotyped, our mean rate of success for genotyping was $>98 \%$. Primers and probes used for the SNP genotyping are available on request.

\section{Statistical genetic analysis}

We performed association analysis in our complex pedigree-based data using the measured genotype approach (MGA) within the variance components (VC) analytical framework implemented in the program SOLAR (http:// www.sfbr.org/solar/, last accessed in July 2006) [35]. The
VC-based approach accounts for the non-independence among family members. In this analytical technique, VCs are modelled as random effects (e.g. additive genetic effects and random environmental effects), whereas the effects of measured covariates such as age and sex are modelled as fixed effects on the trait mean. The VCs, the association parameters, and the other covariate effects (e.g. age and sex terms) were estimated, simultaneously, by maximum likelihood techniques. A likelihood function based on multivariate normal density was numerically maximised to obtain parameter estimates.

Prior to performing MGA, the quantitative transmission disequilibrium test [36], as implemented in SOLAR, was used to examine hidden population stratification, using the notation of within $(w)$ and between $(b)$ family components. In MGA, generally, the marker genotypes were incorporated in the mean effects model as a measured covariate, assuming additivity of allelic effects [37, 38]. Using the notation of within $(w)$ and between $(b)$ family components, a significant test of $b=w$ vs $b=0$ and $w=0$ reflects a significant difference between the genotypic means (Table 1). All analyses included age and sex terms as covariates, if found to be significant. The tests of association and population stratification and the related hypothesis testing are detailed in Table 1. The nested models were compared using the likelihood ratio test. Twice the difference between the loglikelihoods of these models yields a test statistic that is asymptotically distributed, approximating a $\chi^{2}$ distribution with $1 d f$. A $p$ value $\leq 0.05$ is considered significant. Using SOLAR, linkage disequilibrium (LD) between SNP pairs was estimated using the absolute value of the correlation coefficient $|\rho|$. However, for the purpose of discussion, $r^{2}$ values are reported to describe the pairwise LD patterns.

The Bayesian quantitative trait nucleotide model

The Bayesian quantitative trait nucleotide (BQTN) analytical technique is employed to analyse SNPs to find the responsible nucleotide variants (the QTNs [39, 40]) influencing a given phenotype. Given complete SNP data for a gene, this statistical technique can be used to identify the sequence variants that are either potentially functional or that exhibit the highest disequilibria with such potential functional sites. The BQTN model is a simple extension of the classical variance component model, which aims to disentangle the genetic architecture of a quantitative trait [41]. This technique has been described in detail elsewhere [41].

Bayesian model selection/model averaging

Since a candidate gene may contain a number of SNPs that could generate several possible competing models of QTN action, we employed a Bayesian model selection/model 
Table 1 Association/linkage disequilibrium and population stratification analyses and hypothesis testing

\begin{tabular}{lll}
\hline Test & Hypothesis & Inference \\
\hline $\begin{array}{l}\text { Population } \\
\text { stratification }\end{array}$ & $b, w$ (both parameters are estimated) vs $b=w$ & Significant $b \neq w$ indicates the presence of population stratification \\
MGA & $b=w$ (parameters are estimated to be equal) & Significant $b=w$ refers to significant difference between genotypic means \\
& vs $b=0, w=0$ (both parameters are fixed to 0$)$ & \\
\hline
\end{tabular}

averaging approach to analyse the SNP data simultaneously in order to estimate the probability that each SNP has a direct effect on the phenotype. This technique has been described in detail elsewhere [41, 42].

The BQTN analysis can be extended to analyse the haplotype data. For a given individual, the most probable haplotypes were generated using the program MERLIN [36], as implemented in SOLAR. The BQTN analytical procedures are implemented in the computer program SOLAR [41].

Conditional linkage/QTN analysis

To examine whether the associated $\mathrm{SNP}(\mathrm{s})$ found by the QTN analysis can account for our reported linkage of triglyceride concentrations at chromosome $12 \mathrm{p} 13.31$, which is close to the ADIPOR2 locus (chromosome 12p13.33), we combined the QTN analysis with our identity-by-descent (IBD)-based variance component linkage analysis. If a variant, or set of variants, in the ADIPOR2 gene is responsible for the observed linkage signal, linkage analysis conditional on a fixed-effect MGA of the polymorphism will yield an expected logarithm of the odds ratio in favour of linkage (LOD) score near zero. Alternatively, if the associated polymorphism is in less than complete linkage disequilibrium with the true functional site, linkage analysis will generally yield a non-zero LOD score. This method has been described in detail elsewhere [43].

\section{Results}

The clinical characteristics of the subjects are shown in Table 2. We genotyped 59 SNPs $(A D I P O Q=9, A D I P O R I=12$ and $A D I P O R 2=38)$, and, of these, $38(A D I P O Q=8, A D I$ $P O R 1=6$, and $A D I P O R 2=24)$ were polymorphic and in Hardy-Weinberg equilibrium (HWE) in our dataset. SNPs that were not in HWE were discarded from further analysis. Schema of genomic structure and variants of the ADIPOQ, ADIPOR1 and ADIPOR2 genes are shown in Fig. 1. The minor allele frequencies (MAFs) of the SNPs, categorised by diabetes status, are shown in Table 3. The frequency of the minor alleles varied from less than 1 to $49 \%$. There were no significant associations with type 2 diabetes (Table 3 ).
We performed association analysis using MGA, after finding no statistical evidence of hidden population stratification. The significant results $(p \leq 0.05)$ of the association analyses are summarised in Table 4. Of the eight SNPs examined in the $A D I P O Q$ gene, four exhibited significant associations with several IRS-related phenotypes. The minor allele for SNPs rs4632532 and rs182052 were significantly associated with an increase in BMI ( $p=0.029$ and $p=0.032$ ), FSI $(p=0.023$ and $p=0.026)$ and SS $(p=0.0089$ and $p=0.0084)$, and a decrease in HOMA- $\% \mathrm{~S}(p=0.015$ and $p=0.016$ ). Two other SNPs, rs 266729 and rs 2241767 , were significantly associated with $\mathrm{SS}(p=0.036$ and $p=0.013)$. For all SNPs genotyped in the $A D I P O Q$ gene, the pairwise LD $\left(r^{2}\right)$ ranged from 0 to 0.98 . The highest pairwise LD found among the eight $A D I P O Q$ SNPs was between SNP pair rs4632532-rs182052 $\left(r^{2}=0.98\right)$, followed by SNP pair rs2241766-rs2241767 $\left(r^{2}=0.92\right)$, as shown in Fig. 2a. The average pairwise LD among the $A D I P O Q$ SNPs was 0.37 .

SNP rs 7539542 of $A D I P O R 1$ was significantly associated with BMI, SS and WC $(p=0.025, p=0.047$ and $p=0.0062$, respectively). The minor allele was associated with an increase in BMI, SS and WC, as shown in Table 4. Correlations between SNP pairs were estimated and are shown in Fig. 2b. The pairwise LD in the ADIPOR1 gene $\left(r^{2}\right)$ ranged from 0 to 0.87 . The highest $\mathrm{LD}$ was between SNP pair rs2275737-rs2275738 $\left(r^{2}=0.87\right)$, as shown in Fig. 2b. The average pairwise LD among the ADIPORI SNPs was 0.39 .

Of note, 14 of the 24 ADIPOR2 SNPs were significantly $(p<0.05)$ associated with fasting plasma triglyceride concentrations. The mean triglyceride concentrations per genotype are depicted in Table 4 for the significantly associated SNPs. The majority of the minor alleles, with the exception of rs1029629 and rs12582624, were associated with decreased triglyceride levels. Figure 2c shows the overall pattern of LD in the ADIPOR2 gene, and Table 4 highlights SNPs that were in strong LD with each other. The average proportion of shared variation between SNPs, measured by the pairwise correlation, was 0.31 , with SNPspecific values ranging from 0 to 0.99 . Of particular note, four of these SNPs (rs10848569, rs929434, rs3809266 and rs 12342) were in high pairwise LD $\left(r^{2}=0.99\right)$ and were strongly associated with triglyceride levels $(p=0.00029$, $p=0.00016, p=0.00027$ and $p=0.00021$ ). 
Table 2 Characteristics of the SAFDS subjects without diabetes distributed across 27 families

\begin{tabular}{|c|c|c|c|c|}
\hline \multirow[b]{2}{*}{ Variable } & \multirow[b]{2}{*}{ Sample size } & \multirow[b]{2}{*}{ Mean \pm SD or $\%$} & \multicolumn{2}{|c|}{ Distributional properties of the phenotypes } \\
\hline & & & Skewness & Kurtosis \\
\hline Number & 323 & - & - & - \\
\hline Women & 187 & $58 \%$ & - & - \\
\hline Age (years) & 323 & $38.3 \pm 15.4$ & - & - \\
\hline Fasting specific insulin (pmol/l) & 293 & $138 \pm 86$ & 1.1 & 0.9 \\
\hline Fasting glucose $(\mathrm{mmol} / \mathrm{l})$ & 323 & $4.9 \pm 0.6$ & 0.7 & 1.0 \\
\hline ln $\mathrm{HOMA}-\% \mathrm{~S}$ & 290 & $3.8 \pm 0.7$ & 0.0 & -0.9 \\
\hline ln HOMA-\% $\%$ & 293 & $5.1 \pm 0.5$ & -0.0 & -0.6 \\
\hline ln Triglycerides & 311 & $4.9 \pm 0.6$ & 0.4 & -0.3 \\
\hline HDL cholesterol (mmol/l) & 309 & $1.0 \pm 0.3$ & 0.6 & 0.3 \\
\hline Leptin (ng/ml) & 292 & $22.2 \pm 17.6$ & 1.4 & 1.6 \\
\hline BMI $\left(\mathrm{kg} / \mathrm{m}^{2}\right)$ & 317 & $29.4 \pm 6.6$ & 1.0 & 1.2 \\
\hline Sum of skinfold thickness (mm) & 319 & $174.0 \pm 56.3$ & 0.2 & -0.4 \\
\hline Waist circumference $(\mathrm{mm})$ & 317 & $946 \pm 171$ & 0.7 & 0.5 \\
\hline
\end{tabular}

Given our previous findings of common genetic influences on IRS-related phenotypes, including obesity, insulin resistance and triglyceride concentrations [10], we verified whether adjustment for measures of obesity and insulin resistance may affect the observed patterns of association between variants in ADIPOR 2 and triglyceride concentrations. Thus, we repeated the association analysis of triglyceride and ADIPOR2 SNP data after accounting for the effects of BMI and HOMA-\%S. All of the initial findings were observed again, but with increased levels of significance, most notably for SNP rs929434 ( $p=0.000060)$, with the exception of SNP rs1468491, where the $p$ value changed from 0.026 to 0.032 .

Following the findings of the association analysis of the SNPs in ADIPOR2 using MGA (Table 4), we performed the BQTN analysis using ten of the 24 SNPs in ADIPOR2 to obtain a more plausible model, given the data. The 14 SNPs that exhibited redundancy owing to relatively high pairwise LDs $\left(r^{2}>0.80\right)$ were excluded from the BQTN analysis. The remaining ten non-redundant SNPs resulted in the examination of 1,024 possible additive gene action models. The model with only SNP rs929434 provided the strongest evidence for the observed associations, with estimated posterior probability of $>0.82$. The BQTN analysis was extended to analyse the predicted ADIPOR 2 haplotypes. Of the 35 observed haplotypes, 13 haplotypes had frequencies $>1 \%$, with a cumulative frequency of $89 \%$. The 35 haplotypes generated 59,536 possible models to be examined by BQTN analysis; however, haplotypic information did not improve the association.

To determine whether rs929434 has relevance to our reported linkage of fasting triglyceride concentrations at chromosome 12p13.31 [11], which is close to the ADI POR2 locus (chromosome 12p13.33), we re-analysed the original triglyceride data using information from our recent genome scan data generated by the Centre for Inherited
Disease Research (CIDR). Using the total fasting triglyceride dataset, for which SNP rs929434 data were also available, we found modest evidence for linkage $(\mathrm{LOD}=1.85)$ of plasma triglyceride levels to a genetic location between markers D12S374 and GATA49D12 at 12p13.31. When we re-analysed the data, conditional on SNP rs929434, the LOD score was reduced to 0.98. Thus, about $47 \%$ of the evidence for linkage at $12 \mathrm{p} 13.31$ was explained by the sequence variation at rs 929434 .

\section{Discussion}

We have previously shown that the Mexican American population is at relatively high risk of obesity, type 2 diabetes and the IRS. The genetic variation in candidate genes $A D I P O Q, A D I P O R 1$ and ADIPOR2 could influence variation in such disease conditions and related traits. In the present study we provide evidence that ADIPOR2 polymorphisms are strongly associated with decreased plasma triglyceride levels. Of the polymorphisms that we genotyped in the ADIPOR2 gene, our strongest association was with fasting triglyceride levels, most notably SNPs rs10848569, rs929434, rs3809266 and rs12342, where we observed high pairwise correlation between all SNPs $\left(r^{2}=0.99\right)$. These four SNPs with relatively high MAF of $44.3-45.1 \%$ appear to have substantial influence on variation in circulating triglyceride levels, and the evidence for association continues to be significant after Bonferroni's correction for multiple testing. Given that there is some correlation between phenotypes and between SNPs, the Bonferroni correction is rather conservative. To further investigate the potential functional relevance of the variants in the ADIPOR2 gene, we performed a BQTN analysis, in which we compared all possible models of gene action. 

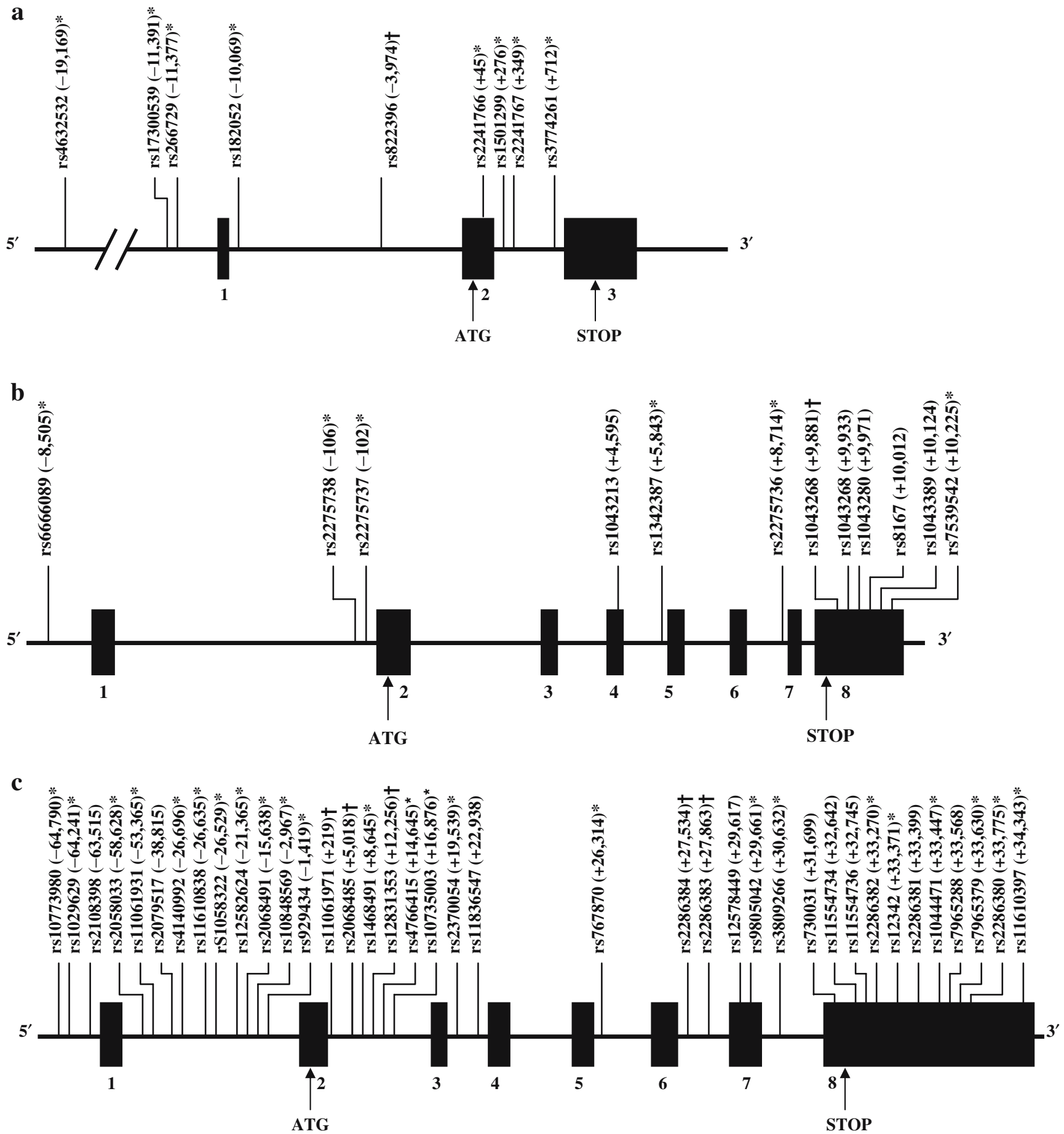

Fig. 1 Genomic structure, and location of SNPs in the ADIPOQ (a), ADIPOR1 (b) and ADIPOR2 (c) genes. Exons are shown as boxes, and introns as lines connecting the boxes. Numbers in parenthesis indicate location relative to the A of the ATG of the start codon. The

This analysis provided strong statistical support for a single SNP model (i.e. rs929434) for the observed association with triglyceride concentrations.

Genetic variants in the $A D I P O R 2$ gene were found to be associated with type 2 diabetes in the Old Order Amish asterisks $(*)$ denote SNPs that were polymorphic in this population $($ MAF $>0.01)$. The SNPs that were not in HWE are denoted by a dagger $(\dagger)$

population [30]. Of the eight SNPs typed (rs10773980, rs1029629, rs11061971, rs10735003, rs9805042, rs12342, rs1044471 and rs2286380), three (rs1029629, rs12342 and rs 1044471) in the Amish population were significantly associated with combined type 2 diabetes and impaired 
Table 3 Minor allele frequencies of SNPs by diabetes status

\begin{tabular}{|c|c|c|c|c|c|}
\hline Gene & SNP rs no. & Position relative to ATG start ${ }^{\mathrm{a}}$ & Major/minor allele & $\begin{array}{l}\text { Frequency \% } \\
\text { (with diabetes) }^{\mathrm{b}}\end{array}$ & $\begin{array}{l}\text { Frequency } \% \\
\text { (without diabetes) }\end{array}$ \\
\hline$A D I P O Q$ & rs4632532 & $-19,169$ & $\mathrm{C} / \mathrm{T}$ & 46.6 & 48.4 \\
\hline$A D I P O Q$ & rs17300539 & $-11,391$ & $\mathrm{C} / \mathrm{T}$ & 3.6 & 2.8 \\
\hline$A D I P O Q$ & rs266729 & $-11,377$ & $\mathrm{C} / \mathrm{G}$ & 33.3 & 30.5 \\
\hline$A D I P O Q$ & rs 182052 & $-10,069$ & $\mathrm{~A} / \mathrm{G}$ & 46.5 & 48.7 \\
\hline$A D I P O Q$ & rs2241766 & 45 & $\mathrm{~T} / \mathrm{G}$ & 10.7 & 10.8 \\
\hline$A D I P O Q$ & rs1501299 & 276 & $\mathrm{G} / \mathrm{T}$ & 28.3 & 29.9 \\
\hline$A D I P O Q$ & rs2241767 & 349 & $\mathrm{~A} / \mathrm{G}$ & 12.7 & 14.9 \\
\hline$A D I P O Q$ & rs3774261 & 712 & $\mathrm{G} / \mathrm{A}$ & 40.7 & 46.8 \\
\hline ADIPOR1 & rs6666089 & $-8,505$ & $\mathrm{G} / \mathrm{A}$ & 15.8 & 19.4 \\
\hline ADIPOR1 & rs 2275738 & -106 & $\mathrm{G} / \mathrm{A}$ & 42.3 & 37.3 \\
\hline ADIPOR1 & rs2275737 & -102 & $\mathrm{C} / \mathrm{A}$ & 41.8 & 40.7 \\
\hline ADIPOR1 & rs1342387 & 5,843 & $\mathrm{~A} / \mathrm{G}$ & 36.5 & 42.4 \\
\hline ADIPOR1 & rs2275736 & 8,714 & $\mathrm{~T} / \mathrm{A}$ & 3.0 & 4.1 \\
\hline ADIPOR1 & rs7539542 & 10,225 & $\mathrm{C} / \mathrm{G}$ & 32.0 & 34.9 \\
\hline ADIPOR2 & rs10773980 & $-64,790$ & $\mathrm{C} / \mathrm{T}$ & 40.4 & 36.5 \\
\hline ADIPOR2 & rs1029629 & $-64,241$ & $\mathrm{~A} / \mathrm{C}$ & 48.9 & 46.6 \\
\hline ADIPOR2 & rs2058033 & $-63,515$ & $\mathrm{C} / \mathrm{A}$ & 21.2 & 24.0 \\
\hline ADIPOR2 & rs11061931 & $-53,365$ & $\mathrm{~T} / \mathrm{A}$ & 6.1 & 6.6 \\
\hline ADIPOR2 & rs4140992 & $-26,696$ & $\mathrm{~T} / \mathrm{C}$ & 35.2 & 32.4 \\
\hline ADIPOR2 & rs11610838 & $-26,635$ & $\mathrm{~T} / \mathrm{C}$ & 6.0 & 7.0 \\
\hline ADIPOR2 & rs1058322 & $-26,529$ & $\mathrm{C} / \mathrm{T}$ & 38.0 & 33.2 \\
\hline ADIPOR2 & rs12582624 & $-21,365$ & $\mathrm{C} / \mathrm{G}$ & 46.6 & 46.5 \\
\hline ADIPOR2 & rs2068491 & $-15,638$ & $\mathrm{~A} / \mathrm{G}$ & 41.0 & 37.9 \\
\hline ADIPOR2 & rs10848569 & $-2,967$ & $\mathrm{~A} / \mathrm{G}$ & 45.7 & 44.3 \\
\hline ADIPOR2 & rs929434 & $-1,419$ & $\mathrm{~A} / \mathrm{G}$ & 45.8 & 45.1 \\
\hline ADIPOR2 & rs1468491 & 8,645 & $\mathrm{C} / \mathrm{G}$ & 4.5 & 5.4 \\
\hline ADIPOR2 & rs4766415 & 12,256 & $\mathrm{~A} / \mathrm{T}$ & 37.1 & 35.4 \\
\hline ADIPOR2 & rs10735003 & 16,876 & $\mathrm{C} / \mathrm{T}$ & 39.8 & 35.9 \\
\hline ADIPOR2 & rs2370054 & 19,539 & $\mathrm{~A} / \mathrm{G}$ & 0 & 0.4 \\
\hline ADIPOR2 & rs767870 & 26,314 & $\mathrm{C} / \mathrm{T}$ & 6.2 & 8.5 \\
\hline ADIPOR2 & rs9805042 & 29,661 & $\mathrm{C} / \mathrm{T}$ & 6.1 & 7.2 \\
\hline ADIPOR2 & rs3809266 & 30,632 & $\mathrm{~T} / \mathrm{G}$ & 45.8 & 44.6 \\
\hline ADIPOR2 & rs2286382 & 33,270 & $\mathrm{G} / \mathrm{A}$ & 6.0 & 7.7 \\
\hline ADIPOR2 & rs 12342 & 33,371 & $\mathrm{~T} / \mathrm{C}$ & 45.8 & 44.3 \\
\hline ADIPOR2 & rs1044471 & 33,447 & $\mathrm{C} / \mathrm{T}$ & 36.2 & 32.6 \\
\hline ADIPOR2 & rs7965379 & 33,630 & $\mathrm{C} / \mathrm{T}$ & 6.1 & 5.9 \\
\hline ADIPOR2 & rs2286380 & 33,775 & $\mathrm{~A} / \mathrm{T}$ & 4.6 & 6.1 \\
\hline ADIPOR2 & rs11610397 & 34,343 & $\mathrm{G} / \mathrm{A}$ & 6.8 & 7.4 \\
\hline
\end{tabular}

${ }^{a}$ Numbers indicate location relative to the A of the start codon (ATG), which is +1

${ }^{b}$ The allele frequencies were not significantly $(p<0.05)$ different between the subjects with and without diabetes based on a chi squared test

glucose tolerance, while in the present study, these SNPs were associated with fasting triglyceride concentrations. Interestingly, the minor allele for rs12342 in the present study, which was significantly associated with decreased fasting triglyceride, was found to be the major allele in the Amish population. In contrast, a study in a Japanese population demonstrated no association between variants (including five that we examined: rs2058033, rs12582624, rs10848569, rs12342 and rs1044471) in ADIPOR2 and type 2 diabetes mellitus [29]. Likewise, no association between variants (including five that we examined: rs 10773980, rs767870, rs12578449, rs9805042 and rs730031) in ADIPOR2 and glucose and lipid metabolic parameters were found in a European population [31].

We previously reported modest evidence for linkage of fasting triglyceride concentrations to a genetic location on chromosome $12 \mathrm{p}$, which is close to the ADIPOR2 locus (chromosome 12p13.33) [11]. To determine whether rs929434 could account for our linkage signal, we reevaluated linkage on chromosome $12 \mathrm{p}$, conditional on the measured genotype effect. By including rs929434 genotypes as a covariate in the model, the LOD score for fasting 
Table 4 Significant associations between genetic polymorphisms in ADIPOQ, ADIPOR1 and ADIPOR2 genes and IRS-related phenotypes

\begin{tabular}{|c|c|c|c|c|c|c|c|}
\hline Gene & SNP rs no. & Phenotype & Major/major ${ }^{\mathrm{a}}$ & Major/minor ${ }^{\mathrm{a}}$ & Minor/minor ${ }^{\mathrm{a}}$ & Direction of change & $p$ value \\
\hline$A D I P O Q$ & rs 4632532 & BMI $\left(\mathrm{kg} / \mathrm{m}^{2}\right)$ & $28.8 \pm 0.9$ & $30.0 \pm 0.6$ & $31.3 \pm 0.8$ & $\uparrow$ & 0.029 \\
\hline$A D I P O Q$ & rs182052 & BMI $\left(\mathrm{kg} / \mathrm{m}^{2}\right)$ & $28.8 \pm 0.9$ & $30.1 \pm 0.6$ & $31.4 \pm 0.8$ & $\uparrow$ & 0.032 \\
\hline$A D I P O Q$ & rs 4632532 & FSI & $108.7 \pm 10.4$ & $126.1 \pm 6.7$ & $143.5 \pm 10.0$ & $\uparrow$ & 0.023 \\
\hline$A D I P O Q$ & rs 182052 & FSI & $108.8 \pm 10.3$ & $125.9 \pm 6.6$ & $143.0 \pm 10.0$ & $\uparrow$ & 0.026 \\
\hline$A D I P O Q$ & rs 4632532 & HOMA-\%S & $3.96 \pm 0.08$ & $3.81 \pm 0.05$ & $3.67 \pm 0.08$ & $\downarrow$ & 0.015 \\
\hline$A D I P O Q$ & rs 182052 & HOMA-\%S & $3.96 \pm 0.08$ & $3.81 \pm 0.05$ & $3.67 \pm 0.08$ & $\downarrow$ & 0.016 \\
\hline$A D I P O Q$ & rs4632532 & $\mathrm{SS}(\mathrm{mm})$ & $140.3 \pm 7.1$ & $151.5 \pm 5.5$ & $162.7 \pm 6.8$ & $\uparrow$ & 0.0089 \\
\hline$A D I P O Q$ & rs 182052 & $\mathrm{SS}(\mathrm{mm})$ & $139.8 \pm 7.1$ & $151.2 \pm 5.5$ & $162.7 \pm 6.8$ & $\uparrow$ & 0.0084 \\
\hline$A D I P O Q$ & rs266729 & $\mathrm{SS}(\mathrm{mm})$ & $157.4 \pm 6.1$ & $147.7 \pm 5.9$ & $137.9 \pm 8.7$ & $\downarrow$ & 0.036 \\
\hline$A D I P O Q$ & rs2241767 & $\mathrm{SS}(\mathrm{mm})$ & $147.8 \pm 5.6$ & $162.2 \pm 6.7$ & $176.6 \pm 11.2$ & $\uparrow$ & 0.013 \\
\hline ADIPOR1 & rs 7539542 & BMI $\left(\mathrm{kg} / \mathrm{m}^{2}\right)$ & $28.8 \pm 1.7$ & $30.5 \pm 1.6$ & $32.2 \pm 1.9$ & $\uparrow$ & 0.025 \\
\hline ADIPOR1 & rs 2275736 & BMI $\left(\mathrm{kg} / \mathrm{m}^{2}\right)$ & $28.9 \pm 1.4$ & $26.6 \pm 1.8$ & $-{ }^{\mathrm{b}}$ & $\downarrow$ & 0.026 \\
\hline ADIPOR1 & rs 7539542 & $\mathrm{SS}(\mathrm{mm})$ & $138.8 \pm 6.7$ & $150.3 \pm 5.9$ & $161.8 \pm 9.4$ & $\uparrow$ & 0.047 \\
\hline ADIPOR1 & rs 7539542 & $\mathrm{WC}(\mathrm{mm})$ & $967.5 \pm 24.3$ & $1020.8 \pm 22.1$ & $1074.0 \pm 33.5$ & $\uparrow$ & 0.0062 \\
\hline ADIPOR2 & $\operatorname{rs} 10773980^{c}$ & $\ln \mathrm{TG}$ & $5.27 \pm 0.07$ & $5.16 \pm 0.06$ & $5.06 \pm 0.08$ & $\downarrow$ & 0.030 \\
\hline ADIPOR2 & rs $1029629^{d}$ & $\ln \mathrm{TG}$ & $5.08 \pm 0.07$ & $5.20 \pm 0.06$ & $5.31 \pm 0.07$ & $\uparrow$ & 0.016 \\
\hline ADIPOR2 & rs4140992 & $\ln \mathrm{TG}$ & $5.25 \pm 0.06$ & $5.14 \pm 0.06$ & $5.03 \pm 0.08$ & $\downarrow$ & 0.019 \\
\hline ADIPOR2 & $\operatorname{rs} 12582624^{d}$ & $\ln \mathrm{TG}$ & $5.06 \pm 0.07$ & $5.19 \pm 0.06$ & $5.31 \pm 0.07$ & $\uparrow$ & 0.0044 \\
\hline ADIPOR2 & $\operatorname{rs} 10848569^{e}$ & $\ln \mathrm{TG}$ & $5.33 \pm 0.07$ & $5.17 \pm 0.05$ & $5.02 \pm 0.07$ & $\downarrow$ & 0.00029 \\
\hline ADIPOR2 & rs929434 $4^{\mathrm{e}}$ & $\ln \mathrm{TG}$ & $5.34 \pm 0.07$ & $5.18 \pm 0.05$ & $5.02 \pm 0.07$ & $\downarrow$ & 0.00016 \\
\hline ADIPOR2 & $\operatorname{rs} 1468491^{\mathrm{f}}$ & $\ln \mathrm{TG}$ & $5.21 \pm 0.06$ & $4.99 \pm 0.10$ & $4.77 \pm 0.19$ & $\downarrow$ & 0.026 \\
\hline ADIPOR2 & rs $4766415^{c}$ & $\ln \mathrm{TG}$ & $5.25 \pm 0.06$ & $5.14 \pm 0.06$ & $5.03 \pm 0.08$ & $\downarrow$ & 0.020 \\
\hline ADIPOR2 & rs $10735003^{c}$ & $\ln \mathrm{TG}$ & $5.28 \pm 0.07$ & $5.17 \pm 0.06$ & $5.05 \pm 0.08$ & $\downarrow$ & 0.016 \\
\hline ADIPOR2 & rs767870 & $\ln \mathrm{TG}$ & $5.22 \pm 0.06$ & $5.01 \pm 0.09$ & $4.80 \pm 0.17$ & $\downarrow$ & 0.013 \\
\hline ADIPOR2 & rs $3809266^{\mathrm{e}}$ & $\ln \mathrm{TG}$ & $5.32 \pm 0.07$ & $5.17 \pm 0.05$ & $5.01 \pm 0.07$ & $\downarrow$ & 0.00027 \\
\hline ADIPOR2 & $\mathrm{rs} 12342^{\mathrm{e}}$ & $\ln \mathrm{TG}$ & $5.33 \pm 0.06$ & $5.17 \pm 0.05$ & $5.02 \pm 0.07$ & $\downarrow$ & 0.00021 \\
\hline ADIPOR2 & rs 1044471 & $\ln \mathrm{TG}$ & $5.26 \pm 0.06$ & $5.15 \pm 0.06$ & $5.05 \pm 0.08$ & $\downarrow$ & 0.027 \\
\hline ADIPOR2 & $\mathrm{rs} 2286380^{\mathrm{e}}$ & $\ln \mathrm{TG}$ & $5.21 \pm 0.06$ & $5.02 \pm 0.09$ & $4.83 \pm 0.17$ & $\downarrow$ & 0.028 \\
\hline ADIPOR2 & rs2058033 & HOMA- $\% \mathrm{~S}$ & $3.75 \pm 0.06$ & $3.89 \pm 0.06$ & $4.02 \pm 0.11$ & $\uparrow$ & 0.046 \\
\hline
\end{tabular}

$T G$ Fasting triglyceride concentrations

${ }^{a}$ Mean trait concentrations by genotype category, after adjusting for age and sex effects, using the measured genotype approach [41]

${ }^{b}$ No minor/minor homozygotes are observed in our model

${ }^{c}$ Three markers are in strong LD with each other $\left(r^{2} \geq 0.92\right)$

${ }^{d}$ Two markers are in strong LD with each other $\left(r^{2}=0.93\right)$

${ }^{e}$ Four markers are in strong LD with each other $\left(r^{2}=0.99\right)$

${ }^{f}$ Two markers are in strong LD with each other $\left(r^{2}=0.99\right)$

triglyceride concentrations was reduced from 1.85 to 0.98 . Thus, about $47 \%$ of the evidence for linkage at $12 \mathrm{p} 13.31$ was explained by the sequence variation at rs 929434 , in turn suggesting that this may be one of several functional variants or in strong LD with the true functional variant(s). The majority of the minor alleles for the polymorphisms in ADIPOR2 were associated with decreased fasting triglyceride and, thus, may provide some protection against developing the IRS and atherosclerosis. Two ADIPOR2 SNPs, rs1029629 and rs12582624, that were in strong LD with each other $\left(r^{2}=0.93\right)$, were associated with increased fasting triglyceride. This seemingly paradoxical finding is not unexpected, given that independent variants in the same gene may have independent effects on traits.

The molecular mechanisms by which adiponectin exerts its insulin-sensitising effects on liver and muscle appear to be related to an increase in fatty acid oxidation, an effect that is mediated, at least in part, by its activation of AMPK [23]. In skeletal muscle, adiponectin acts through its receptors (ADIPOR1 and ADIPOR2) to phosphorylate AMPK, which, in turn, phosphorylates acetyl CoA carboxylase. This leads to an increase in fatty acid oxidation [22, 23 ] and a reduction in toxic intracellular lipid metabolites that inhibit insulin signalling. Adiponectin also decreases hepatic glucose output by reducing the expression of enzymes involved in gluconeogenesis in the liver [44, 45], thereby contributing to the regulation of whole-body glucose homeostasis. As a result of these processes, a reduction in tissue triglycerides and toxic lipid metabolites is observed in both skeletal muscle and liver [19, 22, 23], and this contributes to improved insulin signal transduction. The effects of adiponectin on muscle and liver were recently reviewed elsewhere [46], and it was suggested that liver is the primary site of adiponectin bioactivity. 


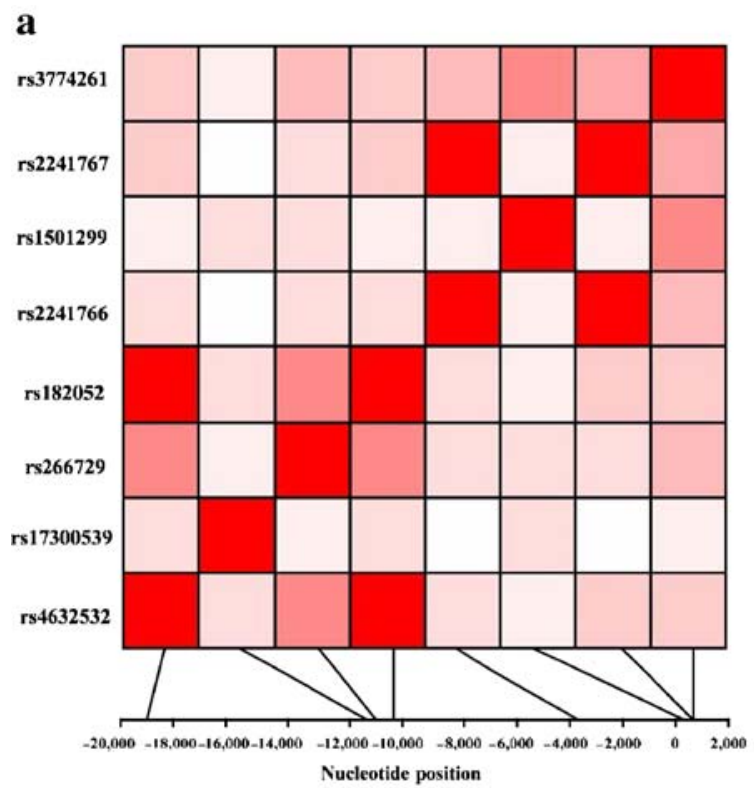

b

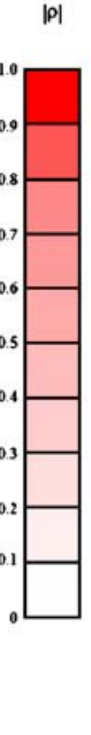

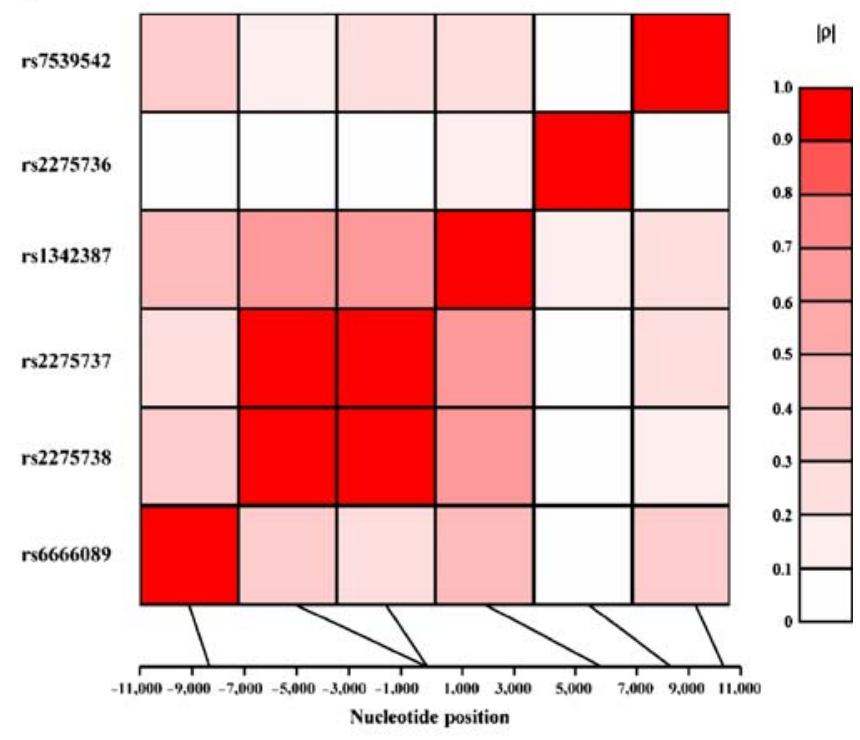

c

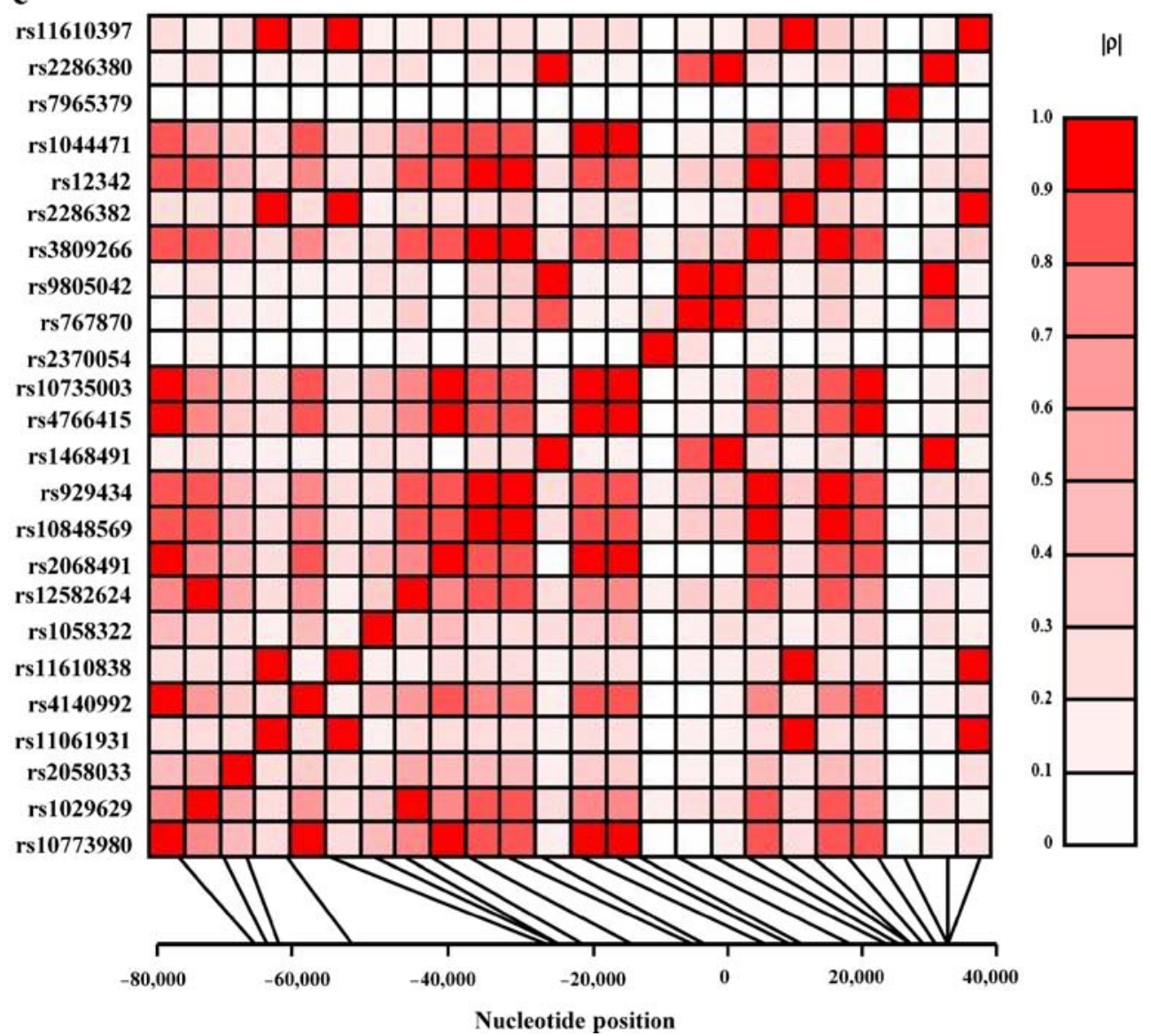

Fig. 2 Linkage disequilibrium (LD) between SNP pairs within $A D I P O Q$ (a), ADIPOR1 (b) and ADIPOR2 (c) genes. SNPs are labelled on the $y$-axis, and the locations (bp) within the gene are shown on the $x$-axis. Pairwise LD is estimated using the correlation coefficient $|\rho|$, and depicted in the figure by the colour intensity of the shaded box, as shown in the legend. The diagonal represents a comparison of each SNP against itself (i.e. $|\rho|=1.0$ ) 
ADIPOR2 is abundantly expressed in human liver [20], and an important question raised by our results is whether adiponectin acts through the hepatic ADIPOR2 to influence triglyceride biosynthesis and homeostasis.

There has been considerable interest in the role of kinetic defects in triglyceride-rich lipoprotein (TRL) metabolism in the development of atherosclerosis and dyslipidaemia (e.g. increased fasting triglycerides and low HDL cholesterol), as it relates to type 2 diabetes and the IRS [47, 48]. It has been suggested that adiponectin concentration may play an independent role in regulating TRL metabolism, aside from its link with abdominal fat, insulin resistance and dyslipidaemia [47]. These observations have relevance to a recent finding where a significant correlation was demonstrated between the expression of ADIPOR1 and ADIPOR 2 mRNA in human skeletal muscle and in vivo parameters of glucose and lipid metabolism [49]. ADIPOR2 mRNA levels correlated positively and independently only with fasting triglyceride concentrations [49], whereas ADIPOR1 mRNA expression was positively correlated with serum insulin and C-peptide concentrations, first-phase insulin secretion, and elevated plasma triglyceride and cholesterol concentrations [49]. Given this independent association of ADIPOR2 gene expression with fasting triglyceride concentrations, but not with BMI, insulin or glucose, and our previous findings of common genetic influences on IRS-related phenotypes (e.g. obesity, insulin resistance and fasting triglyceride) [10], we examined whether adjustment for measures of obesity and insulin resistance may affect the observed patterns of association between variants in ADIPOR2 and fasting triglyceride. The sample size was reduced $(N=273)$ because of the requirement of common covariate information. All the initial findings were observed again, but with increased levels of significance (with the exception of SNP rs1468491, as noted above). For example, the $p$ value for association of SNP rs929434 with fasting triglyceride concentrations was 0.000060 . These results, together with previous findings [49], indicate that adiponectin and its receptors, especially $A D I P O R 2$, play an important role in regulating fasting plasma triglyceride levels, and that they could have a potential role in VLDL metabolism.

Several studies have shown that the $A D I P O Q$ gene is associated with IRS-related phenotypes [24-27]. Of particular note, a haplotype including two SNPs at positions -11,391 (rs17300539) and $-11,377$ (rs266729), both located in the promoter sequence of the $A D I P O Q$ gene, was shown to be strongly associated with adiponectin concentrations and type 2 diabetes in the French population [50]. Our results provide additional evidence that variants of the $A D I P O Q$ gene are significantly associated with IRS-related traits.

We genotyped 12 SNPs in the ADIPOR1 gene (Fig. 1), and six of these were polymorphic and in HWE in our dataset. Of the six polymorphic SNPs, one was significantly associated with IRS-related traits. Interestingly, SNP rs7539542 of ADIPOR1 was significantly associated with three obesity traits: SS, BMI and waist circumference. Similar to our findings, variants in ADIPOR 1 were found to be associated with type 2 diabetes in the Old Order Amish population [30]. The following five SNPs were evaluated in both studies: rs6666089, rs2275738, rs2275737, rs1342387 and rs7539542. In the Amish population, allele/genotype frequencies of SNPs rs2275737 and rs1342387 differed significantly between subjects with type 2 diabetes $v s$. those with normal glucose tolerance. However, there were no significant associations between ADIPOR1 SNPs and type 2 diabetes, insulin sensitivity or insulin secretion in northern Europeans, African Americans [28] or a Japanese population [29]. In contrast, a recent study in a European population demonstrated significant association between several SNPs (including the two we examined, rs666089 and rs1342387) and insulin sensitivity/ liver fat [31].

In conclusion, this study demonstrates that polymorphisms in ADIPOR1, ADIPOR2 and ADIPOQ are associated with several IRS-related phenotypes in Mexican Americans, including plasma triglyceride levels, SS, BMI and waist circumference. Of particular note, multiple genetic variants in ADIPOR2 were strongly associated with decreased triglyceride levels. Our findings of multiple strong associations between ADIPOR2 polymorphisms and plasma triglyceride concentrations may have important implications for atherogenesis and/or dyslipidaemia, owing to the potential influence of $A D I P O R 2$ genetic variation on triglyceriderich lipoprotein metabolism.

Acknowledgements We thank the participants of SAFDS and are grateful for their participation and cooperation.

Duality of interest We do not have any conflicts of interest with respect to the study.

\section{References}

1. DeFronzo RA (1997) Pathogenesis of type 2 diabetes: metabolic and molecular implications for identifying diabetes genes. Diabetes Rev 5:177-269

2. Bays H, Mandarino L, DeFronzo RA (2004) Role of the adipocyte, free fatty acids, and ectopic fat in pathogenesis of type 2 diabetes mellitus: peroxisomal proliferator-activated receptor agonists provide a rational therapeutic approach. J Clin Endocrinol Metab 89:463-478

3. Reaven GM (1995) Pathophysiology of insulin resistance in human disease. Physiol Rev 75:473-486

4. DeFronzo RA (1997) Insulin resistance: a multifaceted syndrome responsible for NIDDM, obesity, hypertension, dyslipidaemia and atherosclerosis. Neth J Med 50:191-197

5. Groop LC, Bonadonna RC, Del Prato S, Ratheiser K, Zych K, DeFronzo RA (1989) Effect of insulin on oxidative and non- 
oxidative pathways of glucose and FFA metabolism in NIDDM. Evidence for multiple sites of insulin resistance. J Clin Invest 84:205-213

6. Bergman RN, Finegood DT, Kahn SE (2002) The evolution of beta-cell dysfunction and insulin resistance in type 2 diabetes. Eur $\mathrm{J}$ Clin Invest 32:35-45

7. Arner P (2001) Free fatty acids - do they play a role in insulin resistance? Diabetes Obes Metab 3:11-19

8. Groop L, Bonadonna R, Simonson DC, Petrides A, Hasan S, DeFronzo RA (1992) Effect of insulin on oxidative and nonoxidative pathways of glucose and free fatty acid metabolism in human obesity. Am J Physiol 263:79-84

9. McGarry JD (2001) Banting Lecture 2001: dysregulation of fatty acid metabolism in the etiology of type 2 diabetes. Diabetes 51:7-18

10. Duggirala R, Blangero J, Almasy L et al (2001) A major locus for fasting insulin concentrations and insulin resistance on chromosome $6 \mathrm{q}$ with strong pleiotropic effects on obesityrelated phenotypes in nondiabetic Mexican Americans. Am J Hum Genet 68:1149-1164

11. Duggirala R, Blangero J, Almasy L et al (2000) A major susceptibility locus influencing plasma triglyceride concentrations is located on chromosome 15q in Mexican Americans. Am J Hum Genet 66:1237-1245

12. Elbein SC (2002) Perspective: the search for genes for type 2 diabetes in the post-genome era. Endocrinology 43:2012-2018

13. McCarthy M (2003) Growing evidence for diabetes susceptibility genes from genome scan data. Curr Diab Rep 3:159-167

14. Maeda K, Okubo K, Shimomura I, Funahashi T, Matsuzawa Y, Matsubara K (1996) cDNA cloning and expression of a novel adipose specific collagen-like factor, apM1 (Adipose Most abundant Gene transcript 1). Biochem Biophys Res Commun 221:286-289

15. Berg AH, Combs TP, Scherer PE (2002) ACRP30/adiponectin: an adipokine regulating glucose and lipid metabolism. Trends Endocrinol Metab 13:84-89

16. Weyer C, Funahashi T, Tanaka S et al (2001) Hypoadiponectinemia in obesity and type 2 diabetes: close association with insulin resistance and hyperinsulinemia. J Clin Endocrinol Metab 86:1930-1935

17. Bajaj M, Suraamornkul S, Piper P et al (2004) Decreased plasma adiponectin concentrations are closely related to hepatic fat content and hepatic insulin resistance in pioglitazone-treated type 2 diabetic patients. J Clin Endocrinol Metab 89:200-206

18. Heilbronn LK, Smith SR, Ravussin E (2003) The insulinsensitizing role of the fat derived hormone adiponectin. Curr Pharm Des 9:1411-1418

19. Yamauchi T, Kamon J, Waki H et al (2001) The fat-derived hormone adiponectin reverses insulin resistance associated with both lipoatrophy and obesity. Nat Med 7:941-946

20. Yamauchi T, Kamon J, Ito Y et al (2003) Cloning of adiponectin receptors that mediate antidiabetic metabolic effects. Nature 423:762-769

21. Civitarese AE, Jenkinson CP, Richardson DK et al (2004) Adiponectin receptors gene expression and insulin sensitivity in nondiabetic Mexican Americans with or without a family history of Type 2 diabetes. Diabetologia 47:816-820

22. Gil-Campos M, Canete RR, Gil A (2004) Adiponectin, the missing link in insulin resistance and obesity. Clin Nutr 23:963-974

23. Yamauchi T, Kamon J, Minokoshi Y et al (2002) Adiponectin stimulates glucose utilization and fatty-acid oxidation by activating AMP-activated protein kinase. Nat Med 8:1288-1295

24. Nakatani K, Noma K, Nishioka J et al (2005) Adiponectin gene variation associates with the increasing risk of type 2 diabetes in nondiabetic Japanese subjects. Int $\mathrm{J}$ Mol Med 15:173-177
25. Gu HF, Abulaiti A, Ostenson CG et al (2004) Single nucleotide polymorphisms in the proximal promoter region of the adiponectin (APM1) gene are associated with type 2 diabetes in Swedish Caucasians. Diabetes 53:31-S35

26. Zacharova J, Chiasson JL, Laakso M; STOP-NIDDM Study Group (2005) The common polymorphisms (single nucleotide polymorphism [SNP] +45 and SNP +276 ) of the adiponectin gene predict the conversion from impaired glucose tolerance to type 2 diabetes: the STOP-NIDDM trial. Diabetes 54: 893-899

27. Vozarova de Courten B, Hanson RL, Funahashi T et al (2005) Common polymorphisms in the adiponectin gene $A C D C$ are not associated with diabetes in Pima Indians. Diabetes 54:284-289

28. Wang H, Zhang H, Jia Y et al (2004) Adiponectin receptor 1 gene $(A D I P O R 1)$ as a candidate for type 2 diabetes and insulin resistance. Diabetes 53:2132-2136

29. Hara K, Horikoshi M, Kitazato H et al (2005) Absence of an association between the polymorphisms in the genes encoding adiponectin receptors and type 2 diabetes. Diabetologia 48:1307-1314

30. Damcott CM, Ott SH, Pollin TI et al (2005) Genetic variation in adiponectin receptor 1 and adiponectin receptor 2 is associated with type 2 diabetes in the Old Order Amish. Diabetes 54: $2245-2250$

31. Stefan N, Machicao F, Staiger H et al (2005) Polymorphisms in the gene encoding adiponectin receptor 1 are associated with insulin resistance and high liver fat. Diabetologia 48: 2282-2291

32. Stern MP, Gaskill SP, Hazuda HP, Gardner LI, Haffner SM (1983) Does obesity explain excess prevalence of diabetes among Mexican Americans? Results of the San Antonio Heart Study. Diabetologia 24:272-277

33. Stern MP, Mitchell BD, Blangero J et al (1996) Evidence for a major gene for type II diabetes and linkage analyses with selected candidate genes in Mexican-Americans. Diabetes 45:563-568

34. Stern MP, Rosenthal M, Haffner SM, Hazuda HP, Franco LJ (1984) Sex difference in the effects of sociocultural status on diabetes and cardiovascular risk factors in Mexican Americans. The San Antonio Heart Study. Am J Epidemiol 120:834-851

35. Almasy L, Blangero J (1998) Multipoint quantitative trait linkage analysis in general pedigrees. Am J Hum Genet 62:1198-1211

36. Abecasis GR, Cookson WOC, Cardon LR (2000) Pedigree tests of transmission disequilibrium. Eur J Hum Genet 8:545-551

37. Boerwinkle E, Chakraborty R, Sing CF (1986) The use of measured genotype information in the analysis of quantitative phenotypes in man. Ann Hum Genet 50:181-194

38. Almasy L, Blangero J (2004) Exploring positional candidate genes: linkage conditional on measured genotype. Behav Genet 34:173-177

39. Long AD, Lyman RF, Langley CH, Mackay TF (1999) Two sites in the Delta gene region contribute to naturally occurring variation in bristle number in Drosophila melanogaster. Genetics 149:999-1017

40. Phillips PC (1999) From complex traits to complex alleles. Trends Genet 15:6-8

41. Blangero J, Goring HH, Kent JW Jr et al (2005) Quantitative trait nucleotide analysis using Bayesian model selection. Hum Biol 77:541-559

42. Curran JE, Jowett JB, Elliott KS et al (2005) Genetic variation in selenoprotein $\mathrm{S}$ influences inflammatory response. Nat Genet 37:1234-1241

43. Almasy L, Blangero J (2004) Exploring positional candidate genes: linkage conditional on measured genotype. Behav Genet 34:173-177 
44. Combs TP, Berg AH, Obici S, Scherer PE, Rossetti L (2001) Endogenous glucose production is inhibited by the adiposederived protein Acrp30. J Clin Invest 108:1875-1881

45. Berg AH, Combs TP, Du X, Brownlee M, Scherer PE (2001) The adipocyte-secreted protein Acrp30 enhances hepatic insulin action. Nat Med 7:947-953

46. Trujillo ME, Scherer PE (2005) Adiponectin-journey from an adipocyte secretory protein to biomarker of the metabolic syndrome. J Intern Med 257:167-175

47. Chan DC, Watts GF, Ng TW et al (2005) Adiponectin and other adipocytokines as predictors of markers of triglyceride-rich lipoprotein metabolism. Clin Chem 51:578-585
48. Hamsten A, Silveira A, Boquist S et al (2005) The apolipoprotein CI content of triglyceride-rich lipoproteins independently predicts early atherosclerosis in healthy middle-aged men. J Am Coll Cardiol 45:1013-1017

49. Staiger H, Kaltenbach S, Staiger K et al (2004) Expression of adiponectin receptor mRNA in human skeletal muscle cells is related to in vivo parameters of glucose and lipid metabolism. Diabetes 53:2195-2201

50. Populaire C, Mori Y, Dina C et al (2003) Does the -11377 promoter variant of $A P M 1$ gene contribute to the genetic risk for Type 2 diabetes mellitus in Japanese families? Diabetologia $46: 443-445$ 